\title{
Serum iron and transferrin in acute neuroleptic induced akathisia
}

\author{
V O'Loughlin, A C Dickie, K P Ebmeier
}

\begin{abstract}
Thirty acute psychiatric patients were examined prospectively at the beginning of neuroleptic treatment for acute psychotic symptoms and on average 16 days later. Two alternative hypotheses were examined: 1) neuroleptic treatment affects the levels of serum iron and transferrin; 2) acute akathisia developing during the initial few weeks of treatment is associated with low levels of serum iron and transferrin, either initially or at follow up or both. Serum iron levels did not change on repeat measurement, while there was a small, but significant decrease of serum transferrin. There was a significantly greater decrease in iron and transferrin levels in patients with akathisia on follow up compared with non-akathisics. In addition, akathisia ratings were highly correlated with serum transferrin levels on follow up.
\end{abstract}

Two independent studies ${ }^{12}$ have reported an inverse correlation between tardive akathisia and plasma or serum iron levels in chronic psychiatric patients stabilised on neuroleptic drugs. The authors argued that akathisia might therefore be related to D2-receptor hypofunction due to a relative deficit of serum and consequently brain iron. Both studies were cross-sectional and included only patients whose medication had not changed for at least one month. In addition, we examined a rather selected sample of chronic psychiatric inpatients, and felt that a prospective study should be carried out on acutely admitted patients who were either just starting to take neuroleptic treatment or whose medication was being substantially increased on admission. It was predicted that about $20 \%$ would develop symptoms and signs of akathisia. $^{3}$

\section{Patients and methods}

Thirty acute psychotic patients were recruited from subsequent admissions to two acute admission wards of the Royal Cornhill Hospital, Aberdeen. They were assessed as soon as possible after admission and followed up for to two to three weeks after the initial examination. The assessment procedure has recently been described in detail. ${ }^{2}$ Akathisia was defined as a score of $\geqslant 10$ on the akathisia scale used in the two previous studies. ${ }^{12}$

Venous blood samples, for the estimation of serum iron and transferrin were taken following a standardised procedure. Serum iron estimation was carried out using a ferrozine colorimetric method on a Technicon RA-1000 analyser (Technicon Instrument Corp, Tarrytown, New York). ${ }^{4}$ The reference range in our own laboratory is $11-30 \mu \mathrm{mol} / 1$. Transferrin estimation was carried out using the same instrument, with an immunoturbimetric method and a Technicon material as calibrator. 5

Ethical approval was given by the Joint Ethical Committee of the Grampian Health Board and the University of Aberdeen. Informed consent was obtained from all subjects.

\section{Results}

Patients were examined on average (SD) 22.9 (13.6) hours after admission. The follow up examination took part on average $16(4 \cdot 8)$ days after the first. Patients' mean (SD) age was $32.9(12 \cdot 1)$. There were 16 men and 14 women, 17 of the patients were diagnosed as bipolar disorder, manic, 13 as schizophrenia or schizophreniform disorder (DSM III-R). ${ }^{6}$ Clinical and laboratory data at both examinations are presented in the table. Six patients $(20 \%)$ qualified for a diagnosis of akathisia at follow up.

In these six patients serum iron levels were $25.8 \mu \mathrm{mol} / 1$ on admission and $20.2 \mu \mathrm{mol} / 1$ at follow up (paired $t$ test: ns). To detect a decrease of serum iron levels by $5.6 \mu \mathrm{mol} / 1$ $(=25 \cdot 8-20 \cdot 2)$ with a power of 0.8 and $p$-value of 0.05 , given the observed standard deviations and assuming an incidence of akathisia of $20 \%$, about 50 patients would be needed. The comparative values were 21.4 and $23.1 \mu \mathrm{mol} / 1$ for non-akathisics (ns). Serum transferrin levels were $2.8 \mathrm{~g} / 1$ on admission and $2.0 \mathrm{~g} / 1$ at follow up in akathisics $(p=0.006)$ and 2.7 and $2.6 \mathrm{~g} / 1$ for nonakathisics (ns). While there were no group differences for iron and transferrin levels on admission, akathisic patients had significantly lower transferrin levels at follow up ( $t$ test: $\mathrm{p}=0.016)$. There was a significant difference between groups for mean changes over time of iron levels $(p=0.0028)$ and transferrin levels 
Table 1 Clinical and laboratory data for 30 patients $(S D)$ at original examination and at follow up

\begin{tabular}{|c|c|c|c|}
\hline & Time 1 & Time 2 & $p^{\star}$ \\
\hline $\begin{array}{l}\text { Chlorpromazine units } \\
\text { BPRS } \\
\text { HAS } \\
\text { Simpson and Angus scale } \\
\text { AIMS } \\
\text { Akathisia scale } \\
\text { Serum iron }(\mu \mathrm{mol} / \mathrm{l}) \\
\text { Serum transferrin }(\mathrm{g} / \mathrm{l})\end{array}$ & $\begin{array}{r}70 \cdot 8(82 \cdot 0) \\
31 \cdot 6(10 \cdot 9) \\
9 \cdot 1(6 \cdot 2) \\
2 \cdot 9(4 \cdot 7) \\
1 \cdot 7(4 \cdot 2) \\
2 \cdot 6(1 \cdot 8) \\
22 \cdot 3(7 \cdot 1) \\
2 \cdot 7(0 \cdot 6)\end{array}$ & $\begin{array}{c}540(234 \cdot 7) \\
23 \cdot 8(12 \cdot 6) \\
6 \cdot 9(6 \cdot 5) \\
7 \cdot 0(5 \cdot 6) \\
2 \cdot 1(4 \cdot 1) \\
5 \cdot 3(4 \cdot 1) \\
22 \cdot 5(6 \cdot 0) \\
2 \cdot 5(0 \cdot 6)\end{array}$ & $\begin{array}{l}0.0001 \\
0.0001 \\
0.035 \\
0.0003 \\
0.4283 \\
0.0002 \\
0.8497 \\
0.0333\end{array}$ \\
\hline
\end{tabular}

${ }^{\star}$ paired $t$ test.

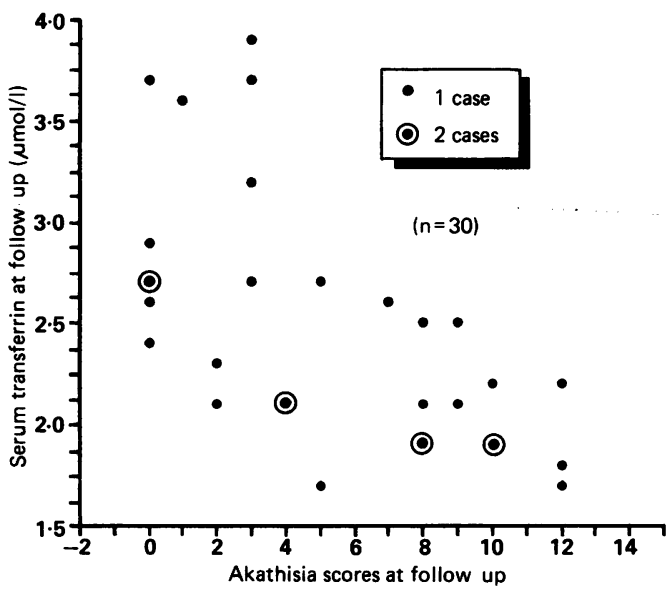

for changes over time in medication, measures of neuroleptic induced movement disorders, apart from akathisia and clinical improvement.

Of the correlations between akathisia scores and iron and transferrin levels at both examinations, only the correlation of transferrin at follow up with akathisia scores at follow up was significant $(\mathrm{r}=0.61, \mathrm{df}=28$, $\mathrm{p}=0.0004)$. Transferrin and iron levels were significantly correlated with each other at the time of first examination $(r=0.44$, $\mathrm{p}=0.0146)$ and at follow up $(\mathrm{r}=0.66$, $\mathrm{p}=0.0001)$. There was no correlation between Simpson and Angus scores for neuroleptic induced Parkinson's syndrome ${ }^{7}$ and akathisia scores $(r=0.06)$. Using a cut-off score of $3 / 4$ on the Simpson and Angus scale, no differences between Parkinsonian and nonParkinsonian patients were found for mean changes of iron ( $t$ test: $p=0 \cdot 1827)$ and transferrin levels $(p=0.7503)$.

\section{Discussion}

As the table shows, we were successful in recruiting an acutely treated group of psychotic patients whose neuroleptic medication increased between examinations, together with Parkinsonian side effects and akathisia scores, whereas psychiatric symptom scores decreased. Across all patients there was a small but significant decrease of transferrin levels, whereas iron levels stayed the same. This overall effect hides, however, a significant difference between groups, in that both iron and, to a larger extent, transferrin levels decreased significantly only in the akathisic patients. It appears, moreover, that patients developing akathisia during the first two weeks of admission are not characterised by initially low iron or transferrin levels. This finding is supported by the inverse correlation between akathisia scores and transferrin levels at follow up (figure), but not on admission.

Although this study is correlational and cannot lead to conclusions about causal relationships, it is tempting to explore possible mechanisms which would explain our findings. Serum transferrin concentrations are regulated in vitro in chorioid plexus epithelial
Figure Transferrin levels plotted against akathisia scores at follow up $(n=30)$.

cells by serotonin in that both transferrin and transferrin mRNA concentrations increase in response to it. ${ }^{8}$ This could provide a rationale 5HT-2 receptor blockers. A differential effect on transferrin levels of patients who do and those who do not develop akathisia would require a difference in this regulation mechanisms between subjects. Transferrin is the main transporter protein for iron in the human body and is involved in the regulation of iron metabolism in the human brain, ${ }^{9}$ so that a reduction of transferrin levels might lead to a relative reduction of the availability of iron to a number of brain regions. As discussed in detail in a previous paper, ${ }^{2}$ iron depletion has been found to mimic D-2 receptor blockade in a variety of animal models and leads to a reversible reduction of $B_{\max }$ for ${ }^{3} \mathrm{H}$ Spiperone binding in the caudate nucleus. A relative lack of iron due to decreased transporter protein in combination with the acute receptor blockade by neuroleptics might therefore combine to produce akathisia in some patients.

1 Brown KW, Glen SE, White T. Low serum iron status and kathisia. Lancet $1987 ; \mathbf{i}: 1234-36$.

2 Barton A, Bowie J, Ebmeier K. Low plasma iron status and akathisia. J Neurol Neurosurg Psychiatry 1990;53:671-4.

3 Braude W, Barnes T, Gore S. Clinical characteristics of akathisia. B J Psychiatry 1983;143:139-50.

4 Stookey L. Ferrozine-A new spectrophotometric reagent for iron. Anal Chem 1970;42:779.

5 Eckman I, Robbins JB, Van den Hamer CJA, Lentz J, Scheinberg IH. Automation of a quantitative immunochemical microanalysis of human serum transferrin: chemical microanalysis of human serum

6 American Psychiatric Association. Diagnostic and Statistical Manual of Mental Disorders, 3rd Ed (Revised). Washing ton DC; American Psychiatric Association, 1987.

8 Tsutsumi M, Skinner MK, Sanders-Bush E. Transferri gene suppression and synthesis by cultured chorioid plexus epithelial cells. Regulation by serotonin and cyclic 265(16): $9626-31$.

9 Gerber MR, Connor JR. Do oligodendrocytes mediate iron regulation in the human brain? Ann Neurol 1989, 26(1):95-8. for an effect of neuroleptics, many of which are

7 Simpson G, Angus J. A rating scale for extrapyramidal side effects. Acta Psychiat Scand 1970;212(Suppl):11-19. adenosine 3'5'-monophosphate. J Biol Chem 1989, 